\title{
Insulin resistance is an independent correlate of high serum levels of advanced glycation end products (AGEs) and low testosterone in non-diabetic men
}

\author{
Nobuhiro Tahara,' Tsutomu Imaizumi,' Masayoshi Takeuchi² and Sho-ichi Yamagishi3,** \\ 'Department of Medicine; Division of Cardio-Vascular Medicine; and ${ }^{3}$ Department of Pathophysiology and Therapeutics of Diabetic Vascular Complications; \\ Kurume University School of Medicine; Kurume; '²Department of Pathophysiological Science; Faculty of Pharmaceutical Science; Hokuriku University; Kanazawa, Japan
}

Key words: AGEs, testosterone, insulin resistance

\begin{abstract}
Advanced glycation end products (AGEs) are involved in cardiovascular disease. Low testosterone level is associated with increased risks of cardiometabolic disorders as well. However, which anthropometric and metabolic variables, including AGEs, are independently correlated with low testosterone is largely unknown. In this study, we investigated whether high serum level of AGEs is one of the independent determinants of low testosterone in non-diabetic men. One hundred thirteen non-diabetic men who did not receive any drugs for hypertension and dyslipidemia underwent a complete history and physical examination, determination of blood chemistries, including serum AGEs and testosterone. Univariate analysis showed that testosterone levels were associated with waist circumference (inversely), diastolic blood pressure (BP) (inversely), mean BP (inversely), triglycerides (inversely), HDL-cholesterol, fasting plasma glucose (inversely), fasting insulin (inversely), homeostasis model assessment of insulin resistance (HOMA-IR) (inversely), AGEs (inversely) and uric acid (inversely). By the use of multiple stepwise regression analyses, HOMA-IR $(p=0.005)$ and triglycerides levels $(p<0.05)$ remained significant and were independently related to testosterone levels $\left(R^{2}=0.168\right)$. HOMA-IR index was one of the independent determinants of serum levels of AGEs as well. The present study demonstrated for the first time that HOMA-IR was independently associated with high serum levels of AGEs and low testosterone in non-diabetic men. Insulin resistance could link elevation of AGEs to testosterone deficiency in non-diabetic men.
\end{abstract}

\section{Introduction}

Reducing sugars can react non-enzymatically with the amino groups of proteins to form reversible Schiff bases and then Amadori products. ${ }^{1-3}$ These early glycation products undergo further complex reactions such as rearrangement, dehydration and condensation to become irreversibly cross-linked, heterogeneous fluorescent derivatives termed "advanced glycation end products" (AGEs). ${ }^{1-3}$ The formation and accumulation of AGEs have been known to progress during normal aging process and at an accelerated rate under hyperglycemic and/or oxidative stress conditions. ${ }^{1-3}$ There is a growing body of evidence to show that AGEs elicit oxidative stress generation and subsequently evoke inflammatory and thrombogenic reactions, thus being implicated in various devastating disorders such as cardiovascular disease, neurodegenerative disorders, cancers, non-alcoholic steatohepatitis and vascular complications in diabetes. ${ }^{4-9}$

As men grow older, serum testosterone levels decline and decreased testosterone level is associated with increased risks of metabolic syndrome, type 2 diabetes, osteoporosis, impaired sexual health and cardiovascular disease. ${ }^{10-14}$ Further, recently, low testosterone level has been shown to predict higher overall and cardiovascular-related mortality in middle-aged and older men as well. ${ }^{13}$ Since some interventional studies indicate that testosterone substitution may be helpful in preventing the metabolic syndrome in aging men, ${ }^{10-14}$ low testosterone could be a therapeutic target for various cardiometabolic disorders. However, which anthropometric and metabolic variables, including AGEs, are independently correlated with low testosterone in men is largely unknown. In this study, we investigated whether high serum level of AGEs is one of the independent determinants of low testosterone in non-diabetic men.

\section{Results}

Backgrounds of the subjects are presented in Table 1 . Mean serum AGEs and testosterone levels were $9.17 \pm 2.69 \mathrm{U} / \mathrm{mL}$ and $4.84 \pm 0.29 \mathrm{ng} / \mathrm{mL}$, respectively. Table 2 shows results 
Table 1. Clinical variables of our subjects

\begin{tabular}{|c|c|}
\hline Clinical variables & \\
\hline Patients (N) & 113 \\
\hline Age (years old) & $58.8 \pm 8.5$ \\
\hline Age (years old) (range) & $39-82$ \\
\hline Waist circumference $(\mathrm{cm})$ & $87.7 \pm 8.5$ \\
\hline Heart rate (beats/minute) & $63.4 \pm 10.1$ \\
\hline Systolic BP (mmHg) & $131.3 \pm 14.0$ \\
\hline Diastolic BP (mmHg) & $83.6 \pm 9.5$ \\
\hline Mean BP $(\mathrm{mmHg})$ & $99.5 \pm 10.5$ \\
\hline Total-cholesterol (mg/dL) & $210.1 \pm 35.7$ \\
\hline LDL-cholesterol (mg/dL) & $125.8 \pm 32.8$ \\
\hline HDL-cholesterol (mg/dL) & $53.6 \pm 12.7$ \\
\hline Triglycerides (mg/dL) & $125.3 \pm 7.5$ \\
\hline Fasting plasma glucose (mg/dL) & $101.4 \pm 6.1$ \\
\hline Fasting Insulin $(\mu \mathrm{U} / \mathrm{mL})$ & $5.40 \pm 0.32$ \\
\hline HOMA-IR & $1.35 \pm 0.08$ \\
\hline $\mathrm{HbA1c}(\%)$ & $5.32 \pm 0.32$ \\
\hline AGEs $(\mathrm{U} / \mathrm{mL})$ & $9.17 \pm 2.69$ \\
\hline Testosterone $(\mathrm{ng} / \mathrm{mL})$ & $4.84 \pm 0.29$ \\
\hline Blood urea nitrogen (mg/dL) & $15.2 \pm 3.1$ \\
\hline Creatinine (mg/dL) & $0.82 \pm 0.05$ \\
\hline Creatinine clearance $(\mathrm{mL} / \mathrm{min})$ & $94.0 \pm 22.7$ \\
\hline Uric acid (mg/dL) & $6.22 \pm 1.14$ \\
\hline $\mathrm{Hs}-\mathrm{CRP}(\mathrm{mg} / \mathrm{L})$ & $0.61 \pm 0.04$ \\
\hline Current smoker (N) & 17 \\
\hline
\end{tabular}

$\mathrm{BP}$, blood pressure; LDL-cholesterol, low-density lipoprotein-cholesterol; HDL-cholesterol, high-density lipoprotein-cholesterol; HOMA-IR, homeostasis model assessment of insulin resistance; HbA1c, glycosylated hemoglobin; AGEs, advanced glycation end products; Hs-CRP, high sensitive C-reactive protein; N, number.

of univariate analysis for determinants of testosterone levels. Parameters statistically and significantly related to testosterone levels were waist circumference (inversely), diastolic blood pressure (BP) (inversely), mean BP (inversely), triglycerides (inversely), high-density lipoprotein-cholesterol (HDLcholesterol), fasting plasma glucose (FPG) (inversely), fasting insulin (inversely), homeostasis model assessment of insulin resistance (HOMA-IR) (inversely), AGEs (inversely) and uric acid (inversely). Because these significant parameters were closely correlated with each other, multiple stepwise regression analysis was performed. Finally, HOMA-IR (inversely, $\mathrm{p}=0.005$ ) and triglycerides (inversely, $\mathrm{p}<0.05$ ) remained significant (Table 3) and were independently related to serum testosterone levels $\left(\mathrm{R}^{2}\right.$ $=0.168)$. Further, HOMA-IR index was found to be one of the independent determinants of serum levels of AGEs as well.

\section{Discussion}

In the present study, we demonstrated for the first time that besides metabolic risk factors such as waist circumference, BP, plasma glucose, lipids and uric acids, serum levels of AGEs were correlated
Table 2. Univariate analysis for correlates of serum testosterone

\begin{tabular}{|c|c|c|c|c|}
\hline Parameters & $\beta$ & SE & t-value & p-value \\
\hline Age & 0.049 & 0.002 & 0.518 & 0.605 \\
\hline Waist circumference & -0.349 & 0.001 & -3.926 & 0.000 \\
\hline Heart rate & -0.102 & 0.001 & -1.076 & 0.284 \\
\hline Systolic BP & -0.174 & 0.001 & -1.866 & 0.065 \\
\hline Diastolic BP & -0.214 & 0.001 & -2.313 & 0.023 \\
\hline Mean BP & -0.206 & 0.001 & -2.220 & 0.028 \\
\hline Total-cholesterol & -0.116 & 0.000 & -1.229 & 0.222 \\
\hline LDL-cholesterol & -0.091 & 0.000 & -0.965 & 0.337 \\
\hline Triglycerides $^{\dagger}$ & -0.326 & 0.054 & -3.627 & 0.000 \\
\hline HDL-cholesterol & 0.252 & 0.001 & 2.748 & 0.007 \\
\hline Fasting plasma glucose ${ }^{\dagger}$ & -0.249 & 0.356 & -2.711 & 0.008 \\
\hline Fasting insulin ${ }^{\dagger}$ & -0.359 & 0.047 & -4.048 & 0.000 \\
\hline HOMA-IR ${ }^{\dagger}$ & -0.368 & 0.044 & -4.173 & 0.000 \\
\hline AGEs & -0.190 & 0.005 & -2.038 & 0.044 \\
\hline $\mathrm{HbA1c}$ & -0.100 & 0.043 & -1.061 & 0.291 \\
\hline Blood urea nitrogen & -0.125 & 0.004 & -1.325 & 0.188 \\
\hline Creatinine $^{\dagger}$ & -0.031 & 0.208 & -0.326 & 0.745 \\
\hline Creatinine clearance & -0.169 & 0.001 & -1.808 & 0.073 \\
\hline Uric acid & -0.258 & 0.011 & -2.810 & 0.006 \\
\hline $\mathrm{Hs}^{-\mathrm{CRP}^{+}}$ & -0.085 & 0.024 & -0.903 & 0.369 \\
\hline Current smoking & -0.048 & 0.028 & -0.505 & 0.615 \\
\hline
\end{tabular}

Univariate analysis revealed that testosterone levels were associated with waist circumference (inversely), diastolic blood pressure (BP) (inversely), mean BP (inversely), triglycerides (inversely), high-density lipoprotein-cholesterol (HDL-cholesterol), fasting plasma glucose (inversely), fasting insulin (inversely), homeostasis model assessment of insulin resistance (HOMA-IR) (inversely), AGEs (inversely) and uric acid (inversely). 'Log-transformed value was used. LDL-cholesterol, low-density lipoprotein-cholesterol; HbA1c, glycosylated hemoglobin; $\mathrm{Hs}-\mathrm{CRP}$, high sensitive $\mathrm{C}$-reactive protein; $\beta$, regression coefficients; $\mathrm{SE}$, standard error.

with low testosterone levels in non-diabetic men. In order to exclude the confounders that could affect the relationship between serum levels of AGEs and testosterone, we limited our analysis to non-diabetic men without anti-hypertension (HT) drugs, aspirin, statins or fibrates because (1) it is well known that serum levels of AGEs are significantly elevated in diabetic patients compared to non-diabetic subjects by reflecting cumulative hyperglycemic burden, ${ }^{1-4}(2)$ testosterone levels are also reported to decrease in diabetic patients, ${ }^{15,16}$ and (3) treatments with anti-HT agents, aspirin, statins or fibrates could reduce circulating levels of AGEs in humans. ${ }^{17-20}$

There is accumulating evidence that AGEs play a role in the development and progression of cardiovascular diseases in both animal models and humans. ${ }^{3,4,21-24}$ Indeed, an inhibitor of AGE formation, aminoguanidine treatment was reported to decrease accumulation of AGEs and atherosclerotic plaque area in the thoracic and abdominal aortas in streptozotocin-induced diabetic apolipoprotein E-deficient mice. ${ }^{21}$ Aminoguanidine reduced plaque formation in the aorta of cholesterol-fed rabbits, and there was a correlation between AGE levels and the degree of atheroma in this model. ${ }^{22}$ Further, AGEs have been actually detected within atherosclerotic 
lesions in humans. ${ }^{23}$ We have previously found that serum levels of AGEs are an independent determinant of plasminogen activator inhibitor-1, one of the biomarkers of future cardiovascular events, in non-diabetic general population. ${ }^{25}$ In addition, Kilhovd et al. reported that serum levels of AGEs could predict total, cardiovascular disease and coronary heart disease mortality in non-diabetic subjects, especially women. ${ }^{26}$ These observations suggest that high circulating levels of AGEs may partly explain the increased risk of future cardiovascular events in men with low testosterone.

In this study, serum levels of AGEs were associated with low testosterone levels in univariate, but not multivariate analysis. Insulin resistance could link the elevation of circulating AGE levels to decreased testosterone in our subjects because HOMA-IR index, a marker of insulin resistance was found to be one of the independent determinants of serum levels of testosterone (Table 3) as well as AGEs ( $\mathrm{p}=0.004)$. Although our study was a cross-sectional one and therefore, did not elucidate the causal relationship among serum levels of AGEs, testosterone and insulin resistance, the present study suggests that insulin resistance may be a therapeutic target for preventing cardiovascular disease in non-diabetic men with low testosterone levels. Longitudinal and/or interventional studies are helpful to clarify whether improvement of insulin resistance could decrease circulating levels of AGEs and increase testosterone levels and subsequently reduce the risk of future cardiovascular events in non-diabetic men.

\section{Research Design and Methods}

Subjects. The study involved 113 consecutive male outpatients in Kurume University Hospital with a mean age of $58.8 \pm 8.5$ years old. None of the patients received anti-HT drugs, aspirin, statins or fibrates. We excluded any patients with inflammatory, neoplastic disorders and those who had a recent ( $<3$ months) acute coronary syndrome, stroke and any acute infection. Patients who received oral hypoglycemic agents or insulin injections for the treatment of diabetes mellitus (DM) and/or whose FPG was more than $126 \mathrm{mg} / \mathrm{dL}$ and/ or whose glycosylated hemoglobin (HbAlc) levels were more than $6.5 \%$ were also excluded. All participants gave informed consent. The Ethical Committee for the Clinical Research of Kurume University approved this study.

Data collection. The medical history, use of smoking and alcohol were ascertained by a questionnaire. Smoking was classified as current habitual use or not. Waist circumference was measured as an index of central obesity. BP was measured in the sitting position using an upright standard sphygmomanometer. Vigorous physical activity and smoking were avoided for at least 30 minutes before $\mathrm{BP}$ and resting heart rate measurements.

Blood was drawn from the antecubital vein in the morning for determinations of lipids (total-cholesterol, low density lipoprotein-cholesterol (LDL-cholesterol), triglycerides and HDL-cholesterol), plasma glucose, insulin, HbA1c, blood urea nitrogen, creatinine, uric acid, high-sensitive $\mathrm{C}$-reactive protein (hs-CRP). These blood chemistries were measured with standard methods at a commercially available laboratory.
Table 3. Multiple stepwise regression analysis for correlates of serum testosterone

$\begin{array}{cccc}\text { Characteristics } & \beta & \text { SE } & \text { Significance } \\ \text { HOMA-IR }^{\dagger} & -0.278 & 0.048 & 0.005 \\ \text { Triglycerides }^{\dagger} & -0.201 & 0.058 & 0.042\end{array}$

Multiple stepwise regression analysis revealed that homeostasis model assessment of insulin resistance (HOMA-IR) (inversely, $\mathrm{p}=0.005$ ) and triglycerides (inversely, $\mathrm{p}<0.05$ ) were independently associated with serum testosterone levels. 'Log-transformed value was used. $\beta$, regression coefficients; $\mathrm{SE}$, standard error. $\mathrm{R}^{2}=0.168$.

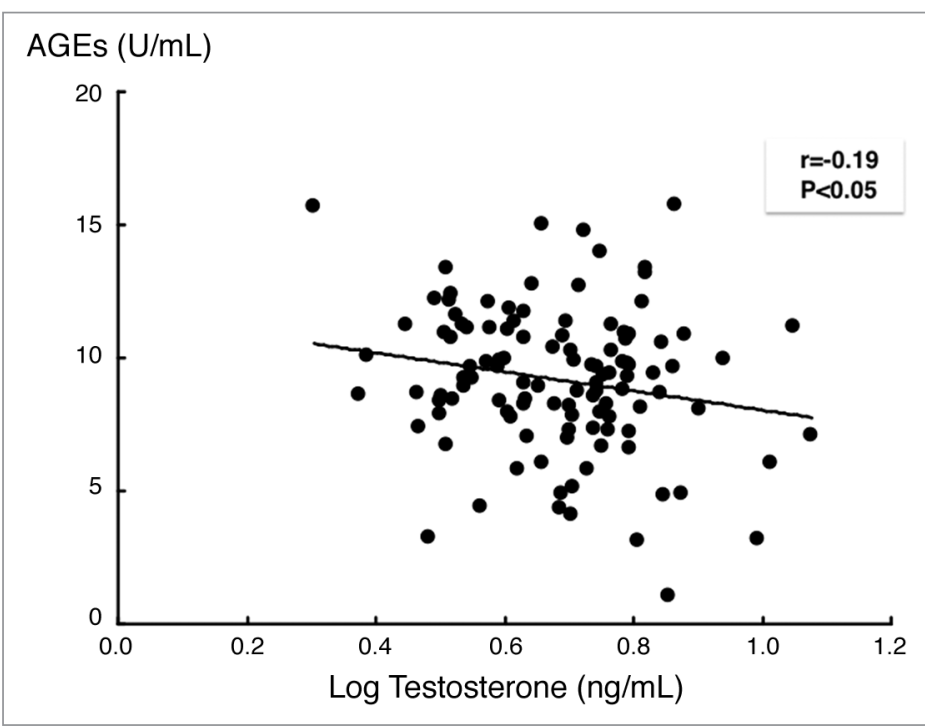

Figure 1. Inverse correlation between serum levels of testosterone and AGEs. Serum levels of AGEs were inversely correlated with testosterone levels in our subjects. One hundred thirteen consecutive male outpatients in Kurume University Hospital with a mean age of $58.8 \pm 8.5$ years old were enrolled in the present study. Blood was drawn from the antecubital vein in the morning. Measurement of AGEs was performed with a competitive enzyme-linked immunosorbent assay (ELISA). In this study, one unit (U) corresponds to $1 \mu \mathrm{g}$ of glyceraldehyde-derived AGE-bovine serum albumin standard. Serum testosterone level was measured with electro-chemiluminescence immunoassay.

(The Kyodo Igaku Laboratory, Fukuoka, Japan) as described previously. ${ }^{27}$ Measurement of AGEs was performed with a competitive enzyme-linked immunosorbent assay (ELISA) as described previously. ${ }^{28}$ In this study, one unit (U) corresponds to $1 \mu \mathrm{g}$ of glyceraldehyde-derived AGE-bovine serum albumin standard. Serum testosterone level was measured with electro-chemiluminescence immunoassay at SRL Inc., (Tokyo, Japan). Insulin resistance was estimated using the HOMA-IR. HOMA-IR index was calculated from the values of FPG $(\mathrm{mg} / \mathrm{dL})$ and fasting insulin $(\mu \mathrm{U} / \mathrm{ml})$ using the following formula [(glucose $\mathrm{x}$ insulin)/405]. Creatinine clearance was calculated with the Cockcroft-Gault equation. ${ }^{29}$

Statistical methods. Because of skewed distributions, the natural logarithmic $(\ln )$ transformations were performed for triglycerides, fasting plasma glucose, fasting insulin, HOMA-IR, testosterone, serum creatinine and hs-CRP. Univariate analysis was performed for determinants of serum testosterone levels. 
Multiple stepwise regression analysis was performed to determine independent determinants of testosterone levels. Statistical significance was defined as $\mathrm{p}<0.05$. All statistical analyses were performed with the use of the SPSS system (SPSS Inc., Chicago, IL).

\section{References}

1. Vlassara H, Bucala R, Striker L. Pathogenic effects of advanced glycosylation: biochemical, biologic and clinical implications for diabetes and aging. Lab Invest 1994; 70:138-51.

2. Brownlee M. Advanced protein glycosylation in diabetes and aging. Ann Rev Med 1995; 46:223-34.

3. Yamagishi S, Imaizumi T. Diabetic vascular complications: pathophysiology, biochemical basis and potential therapeutic strategy. Curr Pharm Des 2005; 11:2279-99.

4. Yamagishi S, Matsui T. Smooth muscle cell pathophysiology and advanced glycation end products (AGEs). Curr Drug Targets 2010; 11:875-81.

5. Yamagishi S, Matsui T. Advanced glycation end products (AGEs), oxidative stress and diabetic nephropathy. Oxid Med Cell Longev 2010; 3:1-8.

6. Yamagishi S, Nakamura K, Matsui T, Ueda S, Fukami $\mathrm{K}$, Okuda $\mathrm{S}$. Agents that block advanced glycation end product (AGE)-RAGE (receptor for AGEs)-oxidative stress system: a novel therapeutic strategy for diabetic vascular complications. Expert Opin Investig Drugs 2008; 17:983-96.

7. Takeuchi M, Yamagishi S. Involvement of toxic AGEs (TAGE) in the pathogenesis of diabetic vascular complications and Alzheimer's disease. J Alzheimers Dis 2009; 16:845-58.

8. Hyogo H, Yamagishi S. Advanced glycation end products (AGEs) and their involvement in liver disease. Curr Pharm Des 2008; 14:969-72.

9. Abe R, Yamagishi S. AGE-RAGE system and carcinogenesis. Curr Pharm Des 2008; 14:940-5.

10. Zitzmann M. Testosterone deficiency, insulin resistance and the metabolic syndrome. Nat Rev Endocrinol 2009; 5:673-81.

11. Yeap BB. Testosterone and ill-health in aging men. Nat Clin Pract Endocrinol Metab 2009; 5:113-21.

12. Kapoor D, Jones TH. Androgen deficiency as a predictor of metabolic syndrome in aging men: an opportunity for intervention? Drugs Aging 2008; 25:357-69.

13. Yeap BB. Are declining testosterone levels a major risk factor for ill-health in aging men? Int J Impot Res 2009; 21:24-36.

\section{Acknowledgements}

This work was supported in part by Grants of Collaboration with Venture Companies Project from the Ministry of Education, Culture, Sports, Science and Technology, Japan (S.Y.). There is no conflict of interest in this paper.

14. Francomano D, Bruzziches R, Natali M, Aversa A Spera G. Cardiovascular effect of testosterone replacement therapy in aging male. Acta Biomed 2010; 81:101-6.

15. Hernández-Mijares A, García-Malpartida K, SoláIzquierdo $\mathrm{E}$, Bañuls $\mathrm{C}$, Rocha M. Testosterone Level in Males with Type 2 Diabetes and Their Relationship with Cardiovascular Risk Factors and Cardiovascular Disease. J Sex Med 2010; In press.

16. Chandel A, Dhindsa S, Topiwala S, Chaudhuri A, Dandona P. Testosterone concentration in young patients with diabetes. Diabetes Care 2008; 31:2013-7.

17. Yamagishi S, Nakamura K, Matsui T. Potential utility of telmisartan, an angiotensin II type 1 receptor blocker with peroxisome proliferator-activated receptor-gamma (PPAR-gamma)-modulating activity for the treatment of cardiometabolic disorders. Curr Mol Med 2007; 7:463-9.

18. Matsui T, Yamagishi S, Takeuchi M, Ueda S, Fukami K, Okuda S. Nifedipine, a calcium channel blocker inhibits advanced glycation end product (AGE)-elicited mesangial cell damage by suppressing AGE receptor (RAGE) expression via peroxisome proliferator-activated receptor-gamma activation. Biochem Biophys Res Commun 2009; 385:269-72.

19. Yamagishi S, Nakamura K, Matsui T. Regulation of advanced glycation end product (AGE)-receptor (RAGE) system by PPAR-gamma agonists and its implication in cardiovascular disease. Pharmacol Res 2009; 60:174-8

20. Li $\mathrm{H}$, Zheng $\mathrm{X}$, Wang $\mathrm{H}$, Zhang $\mathrm{Y}$, Xin $\mathrm{H}$, Chen X. XLF-III-43, a novel coumarin-aspirin compound, prevents diabetic nephropathy in rats via inhibiting advanced glycation end products. Eur J Pharmacol 2010; 627:340-7.

21. Forbes JM, Yee LT, Thallas V, Lassila M, Candido R, Janswlwit-Dahm KA, et al. Advanced glycation end product interventions reduce diabetes-accelerated atherosclerosis. Diabetes 2004; 53:1813-23.
22. Panagiotopoulos S, O'Brien RC, Bucala R, Cooper $\mathrm{ME}$, Jerums G. Aminoguanidine has an anti-atherogenic effect in the cholesterol-fed rabbit. Atherosclerosis 1998; 136:125-31.

23. Nakamura $Y$, Horii $Y$, Nishino T, Shiki H, Sakaguchi $Y$, Kagoshima T, et al. Immunohistochemical localization of advanced glycosylation end products in coronary atheroma and cardiac tissue in diabetes mellitus. Am J Pathol 1993; 43:1649-56.

24. Barlovic DP, Thomas MC, Jandeleit-Dahm K. Cardiovascular disease: what's all the AGE/RAGE about? Cardiovasc Hematol Disord Drug Targets 2010; 10:7-15.

25. Yamagishi S, Adachi H, Takeuchi M, Enomoto $M$ Furuki K, Matsui T, et al. Serum level of advanced glycation end-products (AGEs) is an independent determinant of plasminogen activator inhibitor-1 (PAI-1) in nondiabetic general population. Horm Metab Res 2007; 39:845-8.

26. Kilhovd BK, Juutilainen A, Lehto S, Rönnemaa T, Torjesen PA, Birkeland KI, et al. High serum level of advanced glycation end products predict increased coronary heart disease mortality in nondiabetic women but not in nondiabetic men: a population-based 18-year follow-up study. Arterioscler Thromb Vasc Biol 2005; 25:815-20.

27. Yamagishi S, Adachi H, Abe A, Yashiro T, Enomoto $\mathrm{M}$, Furuki K, et al. Elevated serum levels of pigment epithelium-derived factor in the metabolic syndrome. J Clin Endocrinol Metab 2006; 91:2447-50.

28. Hyogo $\mathrm{H}$, Yamagishi S, Iwamoto $\mathrm{K}$, Arihiro $\mathrm{K}$, Takeuchi M, Sato T, et al. Elevated levels of serum advanced glycation end products in patients with nonalcoholic steatohepatitis. J Gastroenterol Hepatol 2007; 22:1112-9.

29. McAlister FA, Ezekowitz J, Tonelli M, Armstrong PW. Renal insufficiency and heart failure. Prognostic and therapeutic implications from a prospective cohort study. Circulation 2004; 109:1004-9. 


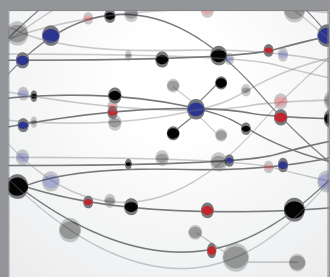

The Scientific World Journal
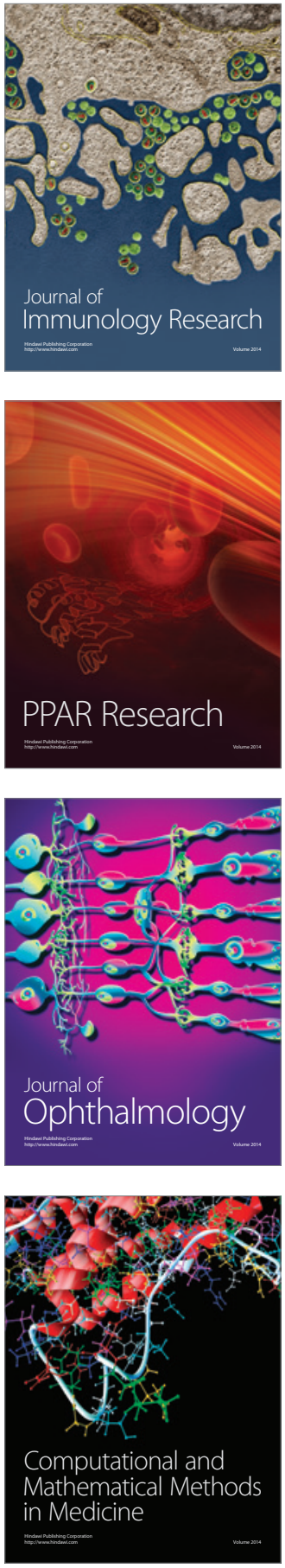

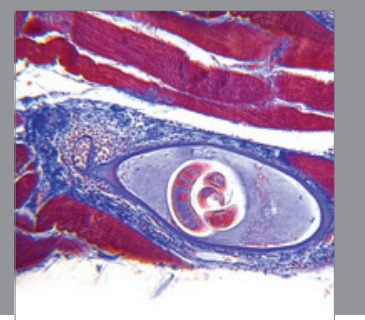

Gastroenterology

Research and Practice
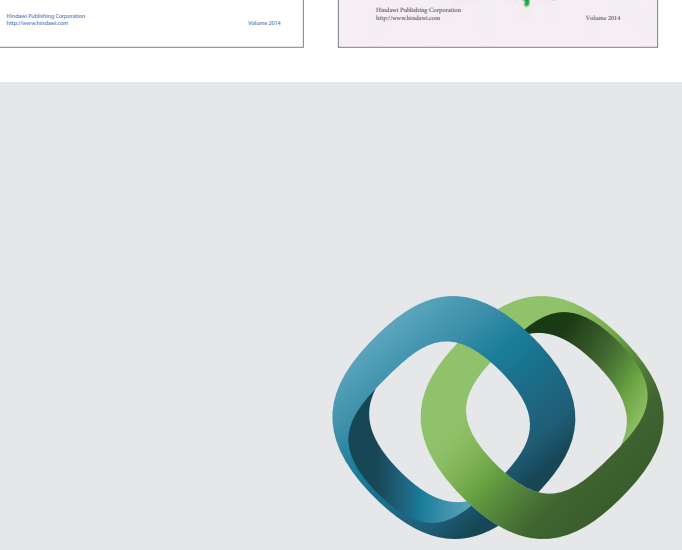

\section{Hindawi}

Submit your manuscripts at

http://www.hindawi.com
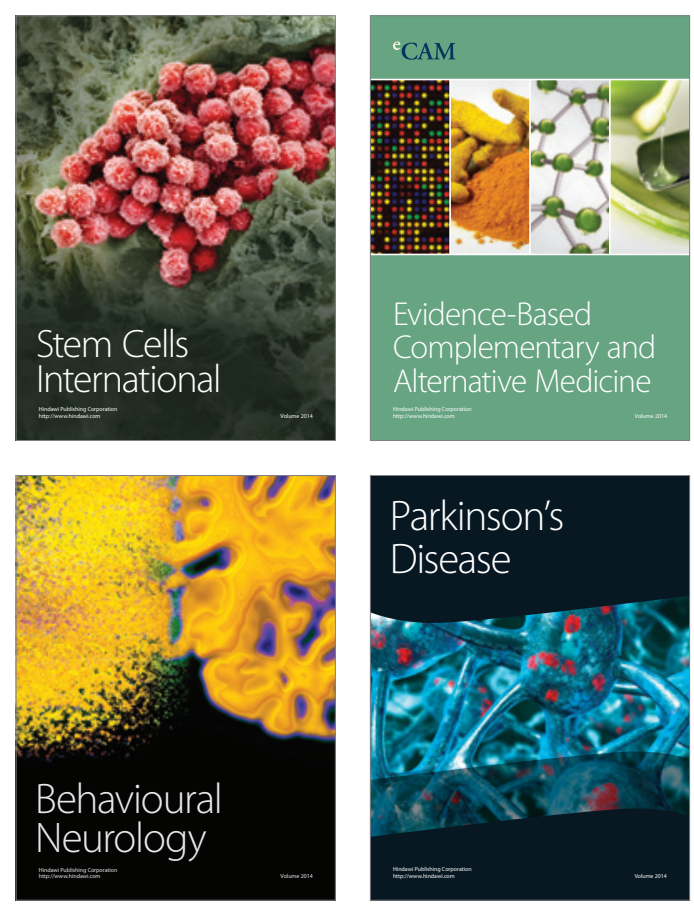

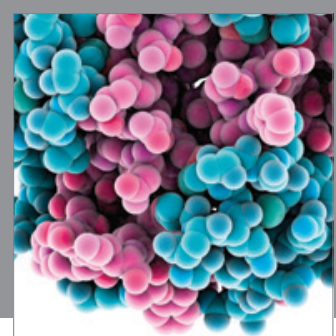

Journal of
Diabetes Research

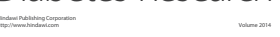

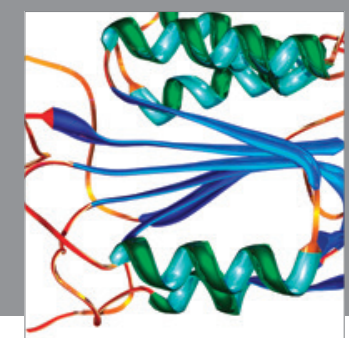

Disease Markers
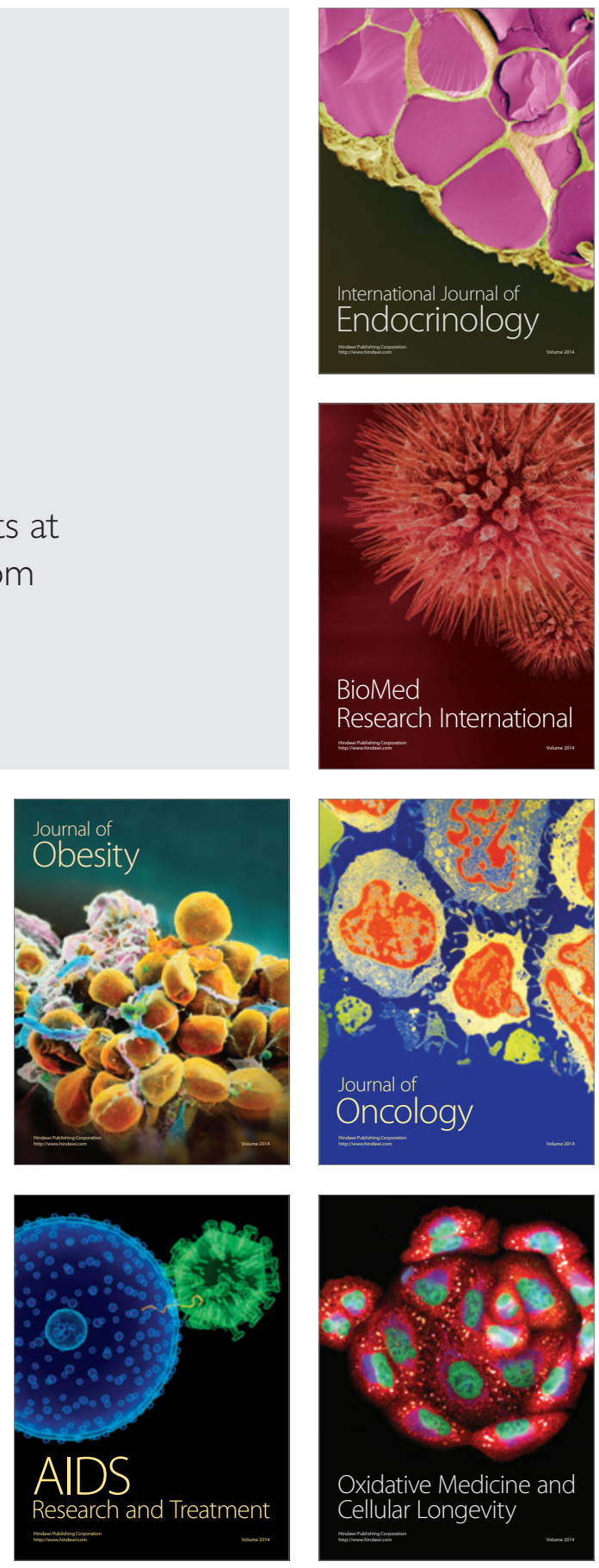